\title{
THE SOLUTION OF AN INTEGRAL EQUATION OF THE FIRST KIND ON A FINITE INTERVAL*
}

BY

\author{
J. A. BELWARD
}

University of Queensland, Australia

1. Introduction. In this paper we shall solve the integral equation

$$
f(x)=\int_{-1}^{1} g(y) K_{0}(\lambda|x-y|) d y, \quad-1<x<1,
$$

where $\lambda$ is a real constant and $K_{0}$ is a modified Bessel function with a logarithmic singularity at the origin which satisfies

$$
x K_{0}^{\prime \prime}(x)+K_{0}^{\prime}(x)-x K_{0}(x)=0,
$$

and has the asymptotic form

$$
K_{0}(x) \sim \pi^{1 / 2}(2 x)^{-1 / 2} e^{-x} \text { as } x \rightarrow \infty .
$$

It will be shown that there exists, for each $n \geq 0$, a pair of functions $f_{n}(x)$ and $g_{n}(x)$ which satisfy Eq. (1.1), the $f_{n}$ 's taking the role of the known function. The $f_{n}$ 's are the Mathieu functions $c e_{n}(\eta)$, where $x=\cos \eta$, and accordingly are complete on $(-1,1)$. Thus for any $f(x)$ belonging to $L_{2}(-1,1)$, the corresponding solution $g(x)$ may be expressed as a series of $g_{n}$ 's.

Equation (1.1) arises from a two-dimensional boundary value problem which provides a description of several physical situations. One well known example of these is that of an ideal fluid of thermal diffusivity $k$ which has a uniform velocity of $2 \lambda k$ past a flat plate fixed tangential to the uniform stream. If the fluid density and thermal constants are assumed independent of temperature and are scaled out of the problem then the temperature of the fluid is given by the solution of

$$
\partial^{2} T / \partial x^{2}+\partial^{2} T / \partial y^{2}-2 \lambda \partial T / \partial x=0
$$

which takes the prescribed values on the plate.

If we put $T=e^{\lambda x} \phi(x, y)$ into (1.2) we obtain

$$
\partial^{2} \phi / \partial x^{2}+\partial^{2} \phi / \partial y^{2}-\lambda^{2} \phi=0
$$

which itself admits a simple physical interpretation. Thus if we replace the convection term $2 \lambda \partial T / \partial x$ in (1.2) by an endothermic release of heat at all points in the fluid proportional to the local temperature then the temperature satisfies Eq. (1.3). Equation (1.3) also occurs in elastic half space problems which have been discussed by Rvachev [1].

If $\phi$ is given at the boundary then we can simulate the effect of the boundary with the distribution of sources along the boundary. The source function for (1.3), that is the

*Received May 31, 1968. 
solution of (1.3) with its right-hand side replaced by $-\delta\left(x-x_{0}\right) \delta\left(y-y_{0}\right)$, is $(2 \pi)^{-1} K_{0}\left(\lambda\left[\left(x-x_{0}\right)^{2}+\left(y-y_{0}\right)^{2}\right]^{1 / 2}\right)$, and if we scatter the sources along the boundary $C$ so that their strength at a point $s$ along the boundary is $g(s)$ then $\phi$ is given by the line integral

$$
\phi\left(x_{0}, y_{0}\right)=\int_{C} g(s) K_{0}\left(\lambda\left[\left(x-x_{0}\right)^{2}+\left(y-y_{0}\right)^{2}\right]^{1 / 2}\right) d s .
$$

If we are given that $\phi=f(x)$ for $y=0,|x| \leq 1$, we obtain the integral equation (1.1) whose solution then gives $\phi$ from (1.4).

There are obvious analytical and computational advantages to be gained from the representation (1.4) and the reduction of the problem to an integral equation in one variable is often an advantage. Conversely a knowledge of the boundary value problem associated with the integral equation (if one exists) can be most helpful-in this case it appears indispensable.

The description of the solution of the integral equation given here follows closely the order in which the problem was finally solved. It was found at an advanced stage of this paper that both Rvachev [1] and Gill [2] had also solved the associated partial differential equation and it appears that Rvachev had the solution of (1.1) within his grasp. Neither author pursued the analysis given here however, and since the equation has received attention from Latta [3] and Pearson [4] it would appear that an explicit solution is of some interest. Further, integral equations of the first kind frequently arise in applied mathematics, and (unless they are of a simple Cauchy type) are often difficult to solve exactly. Thus although this method may not immediately yield the solutions of similar equations it may be of some assistance in establishing the functions appropriate to the problem.

In the context of Eq. (1.1), Latta has introduced an ingenious technique which reduces the problem to an eigenvalue problem in ordinary differential equations, while Pearson has shown that if the kernel of (1.1) is approximated by

$$
P(x) \cdot \log |x|+Q(x)
$$

where $P$ and $Q$ are polynomials, then this approximate equation may be solved exactly.

The present author has attempted to use the method of Lundgren and Chiang [5] on this problem, but their method yields a solution of the form

$$
\left(1-x^{2}\right)^{-1 / 2}\left(a_{0}+a_{1} x+a_{2} x^{2} \cdots\right)
$$

where the $a$ 's can only be determined by substitution into the original equation, a result which has already been established by Latta.

2. Derivation of the solution of the integral equation using an associated boundary value problem. The solution of Eq. (1.1) may be found indirectly by solving the boundary value problem mentioned in the introduction. We shall use this approach to provide the motivation for our choice of a set of complete functions, from which we can generate a solution of Eq. (1.1) for any function $f$ of the class $L_{2}(-1,1)$.

Consider then Eq. (1.1), together with the function

$$
\phi(x, y)=\int_{-1}^{1} g(t) \cdot K_{0}\left(\lambda\left[(x-t)^{2}+y^{2}\right]^{1 / 2}\right) d t .
$$

This function satisfies the partial differential equation 


$$
\partial^{2} \phi / \partial x^{2}+\partial^{2} \phi / \partial y^{2}-\lambda^{2} \phi=0 \text { outside } y=0, \quad|x|<1,
$$

and its behaviour at infinity is described by

$$
\phi(x, y)=O\left(e^{-\lambda r}\right) \quad \text { as } \quad r^{2}=x^{2}+y^{2} \rightarrow \infty .
$$

It is clear that

$$
f(x)=\phi(x, 0+)=\phi(x, 0-), \quad|x|<1,
$$

and we can also show that

$$
g(x)=(1 / 2 \pi)\left[\phi_{y}(x, 0-)-\phi_{y}(x, 0+)\right], \quad|x|<1,
$$

which follows from the representation of the delta function

$$
\lim _{y \rightarrow 0+} \frac{y}{x^{2}+y^{2}}=\pi \delta(x) .
$$

Therefore given $f(x)$, say, Eqs. (2.1a, b, and c) serve to determine $\phi$ and (2.1d) then gives $g(x)$. Similarly, given $g(x)$ we could determine $f(x)$. (This latter case amounts to evaluating the integral on the right-hand side of (1.1) for a given $g(x)$ and, as we shall see, this is not a simple exercise.)

The appropriate coordinates for the solution of this problem are elliptic coordinates and we set

$$
x=\cosh \xi \cos \eta, \quad y=\sinh \xi \sin \eta .
$$

The $x y$ plane then corresponds to $-\pi<\eta \leq \pi$ and $\xi \geq 0$. In terms of these coordinates Eqs. (2.1) transform to

$$
\begin{gathered}
\partial^{2} \Phi / \partial \xi^{2}+\partial^{2} \Phi / \partial \eta^{2}-\left(\lambda^{2} / 2\right)(\cosh 2 \xi-\cos 2 \eta) \Phi=0, \quad-\pi<\eta \leq \pi, \quad \xi \geq 0 \\
\Phi(\xi, \eta)=0\left(\exp \left(-\lambda e^{-\xi}\right)\right) \text { as } \xi \rightarrow \infty, \\
\Phi(0, \eta)=f(\cos \eta) \\
g(\cos \eta)=-\frac{1}{2 \pi|\sin \eta|}\left(\Phi_{\xi}(0+,-\eta)+\Phi_{\xi}(0+, \eta)\right), \quad|\eta|<\pi .
\end{gathered}
$$

Equation (2.3a) is separable in the usual way and after substituting $\Phi=F(\xi) G(\eta)$ we obtain

$$
F_{\xi \xi}-\left(k+\frac{\lambda^{2}}{2} \cosh 2 \xi\right) F=0, \quad G_{\eta \eta}+\left(k+\frac{\lambda^{2}}{2} \cos 2 \eta\right) G=0,
$$

where $k$ is the separation constant.

These are the usual modified Mathieu and Mathieu equations, for which McLachlan [6] and Meixner and Schäfke [7] are standard reference texts.

To obtain solutions of period $2 \pi$ and symmetric in $\eta$, and to satisfy the condition at infinity, we choose values $k_{n}$ of $k$ so that the solutions of $(2.4)$ are $F e k_{n}\left(\xi,-\lambda^{2} / 4\right)$ and $c e_{n}\left(\eta,-\lambda^{2} / 4\right)$.

We will thus fit a solution of the form

$$
\Phi=\sum_{n=0}^{\infty} a_{n} c e_{n}\left(\eta,-\frac{\lambda^{2}}{4}\right) F e k_{n}\left(\eta,-\frac{\lambda^{2}}{4}\right)
$$

to the boundary condition (2.3c). 
Now the $c e_{n}$ 's are complete on $(-\pi, \pi)$ for any even function of $\eta$ and hence if $f(\cos \eta)$ belongs to $L_{2}(-1,1)$ we may expand it in a series of $c e_{n}$ 's. Hence if

$$
f(\cos \eta)=\sum_{n=0}^{\infty} \alpha_{n} c e_{n}\left(\eta,-\frac{\lambda^{2}}{4}\right),
$$

comparison of (2.5) and (2.6) gives

$$
a_{n}=\alpha_{n}\left[F e k_{n}\left(0,-\lambda^{2} / 4\right)\right]^{-1}, \quad n=0,1,2, \cdots
$$

Equation (2.3d) finally gives

$$
g(\cos \eta)=-\frac{1}{\pi|\sin \eta|} \sum_{n=0}^{\infty} \alpha_{n} F e k_{n}^{\prime}\left(0,-\frac{\lambda^{2}}{4}\right)\left[F e k_{n}\left(0,-\frac{\lambda^{2}}{4}\right)\right]^{-1} c e_{n}(\eta)
$$

and this is the solution of (1.1).

So far in this section we have repeated, almost exactly, the analysis given by Rvachev, and, in essence, that given by Gill. Rvachev proceeds using Chebyshev polynomials while Gill's interest centers only on the partial differential equation. Rvachev's main concern lies in a computation of the solution and he does not continue the analysis pursued here.

Now suppose we demand that

$$
f(\cos \eta)=c e_{p}(\eta) .
$$

(Henceforth we shall write $c e_{n}\left(\eta,-\lambda^{2} / 4\right)$ as $c e_{n}(\eta)$ unless we wish to draw particular attention to the parameter, with a similar interpretation for $F e k_{n}(\xi)$.) Then

$$
a_{n} \equiv 0, \quad n \neq p, \quad \text { and } \quad a_{p}=\left[F e k_{p}(0)\right]^{-1}
$$

we have therefore

$$
g(\cos \eta)=\frac{(-1)}{\pi|\sin \eta|} F e k_{p}^{\prime}(0) \cdot\left[F e k_{p}(0)\right]^{-1} c e_{p}(\eta)
$$

and if we substitute this particular result into (1.1), by setting $x=\cos \alpha$, and $y=\cos \beta$ we obtain a very neat result:

$$
\mu_{\nu} \int_{0}^{\pi} \psi_{p}(\beta) K_{0}(\lambda|\cos \alpha-\cos \beta|) d \beta=\psi_{p}(\alpha), \quad 0<\alpha<\pi,
$$

where

$$
\mu_{p}=-F e k_{p}^{\prime}(0)\left[\pi F e k_{p}(0)\right]^{-1}
$$

and

$$
\psi_{p}(\alpha)=\pi^{-1 / 2} 2^{1 / 2} c e_{p}(\alpha) .
$$

We can interpret this result as a formula for the Fourier coeff.cients of $K_{0}(\lambda \mid \cos \alpha-$ $\cos \beta \mid)$ and since $K_{0}(x)$ is analytic for $x \neq 0$, it follows, in the strict classical sense, that $K_{0}(\lambda|\cos \alpha-\cos \beta|)=\sum_{p=0}^{\infty} \frac{\psi_{p}(\alpha) \psi_{p}(\beta)}{\mu_{p}}$, for $\alpha \pm \beta \neq 2 n \pi$,

$$
n=\cdots,-1,0,1,2, \cdots .
$$

Note the close resemblance to Mercer's Theorem for the eigenfunction expansion of a 
continuous kernel with all but a finite number of eigenvalues of one sign. Here the kernel has a weak singularity with arbitrary signed eigenvalues and in this example the conclusions of Mercer's Theorem hold everywhere, except at the singularity, under less stringent conditions.

In the current problem we regard Eq. (2.11) as giving a set of eigenvalues and eigenfunctions from which we can generate the solution of (1.1). The inference we draw from Eq. (2.11) is the following:

Given

$$
\int_{-1}^{1} g(y) K_{0}(\lambda|x-y|) d y=f(x), \quad-1<x<1,
$$

if

$$
f(x)=\sum_{0}^{\infty} \alpha_{n} c e_{n}(\arccos x)
$$

then

$$
g(x)=-\frac{1}{\pi}\left(1-x^{2}\right)^{-1 / 2} \sum_{n=0}^{\infty} \alpha_{n} F e k_{n}^{\prime}(0)\left[F e k_{n}(0)\right]^{-1} c e_{n}(\arccos x) .
$$

We note the expected presence of the factor $\left(1-x^{2}\right)^{-1 / 2}$.

3. A direct verification of Eq. (2.11). The analysis given in Sec. 2 is quite unnecessary for the solution of Eq. (1.1) if we have Eq. (2.11) at our disposal. The most elegant presentation of the solution would simply demonstrate the validity of Eq. (2.11), and Eqs. (2.12) would then follow from the possibility of expanding both known function $f$ and unknown function $g(\eta) \sin \eta$ in terms of a complete set of eigenfunctions. The presence of the factor $\sin \eta\left(=\left(1-x^{2}\right)^{1 / 2}\right)$ can readily be deduced from the form of (2.11). In this section we will verify (2.11) directly.

As mentioned previously, for most functions $g(x)$ it appears impossible to evaluate the right-hand side of (1.1) in terms of known functions (e.g. try $g(x)=1$ ), and it is only from the analysis given in the previous section that we have acquired the foresight to use Mathieu functions. Even with this information the calculations are lengthy and so it might be helpful to the reader if we identify the various stages of the calculation with the equations which follow.

The first step is to express the Bessel function as an integral; from this we obtain a pair of repeated integrals, one of which vanishes according to the parity of the order of the Mathieu function $c e_{p}(\eta)$ (Eqs. (3.1)-(3.3)). We then consider in detail the case of $p$ even commencing with an application of a formula which expresses a modified Mathieu function as an integral involving an ordinary Mathieu function ((3.4) and (3.5)). A second application of this relationship is then used to obtain the Fourier coefficients of $I_{2 m}$ (Eq. (3.7)) whose values are finally determined by evaluating the right-hand side of Eq. (3.8). Further integral relations are used in Eqs. (3.9)-(3.15) and we find that the only nonzero Fourier coefficient of $I_{2 m}$ is the $2 m$ th. It follows (from the orthogonality properties of the $c e_{n}$ 's) that $I_{2 m}(\alpha)$ is proportional to $c e_{2 m}(\alpha)$ and the last step in the manipulations is reached when we show that the constant of proportionality which arises from our verification is that which appears in (2.11) (Eq. 3.17).

We use the following integral representation of the modified Bessel function: 


$$
K_{0}(x)=\int_{0}^{\infty} \cos (x \sinh t) d t, \quad x>0
$$

Let

$$
I_{p}=\int_{0}^{\pi} c e_{p}\left(\beta,-\frac{\lambda^{2}}{4}\right) K_{0}(\lambda|\cos \alpha-\cos \beta|) d \beta ;
$$

then using (3.1) we obtain

$$
I_{p}=\int_{0}^{\pi} c e_{p}\left(\beta,-\frac{\lambda^{2}}{4}\right) d \beta \int_{0}^{\infty} \cos [(\cos \alpha-\cos \beta) \lambda \sinh t] d t .
$$

A permissible inversion of the order of integration and an expansion of the cosine follows and we find

$I_{p}=\int_{0}^{\infty} d t \cos (\lambda \cos \alpha \sinh t) \int_{0}^{\pi} c e_{p}\left(\beta,-\frac{\lambda^{2}}{4}\right) \cos (\lambda \cos \beta \sinh t) d \beta$

$$
+\int_{0}^{\infty} d t \sin (\lambda \cos \alpha \sinh t) \int_{0}^{\pi} c e_{\nu}\left(\beta,-\frac{\lambda^{2}}{4}\right) \sin (\lambda \cos \beta \sinh t) d \beta .
$$

We have to consider separately the cases $p$ odd and $p$ even. First let $p=2 m$; then the inner integral of the second term on the right-hand side of (3.2) equals

$\int_{0}^{\pi} c e_{2 m}\left(\beta,-\frac{\lambda^{2}}{4}\right) \sin (\lambda \cos \beta \sinh t) d \beta=(-1)^{m} \int_{-\pi / 2}^{\pi / 2} c e_{2 m}\left(\beta_{1}, \frac{\lambda^{2}}{4}\right) \sin \left(\lambda \sin \beta_{1} \sinh t\right) d \beta_{1}$ where we have substituted $\beta=\pi / 2-\beta_{1}$ and used the relationship

$$
c e_{2 m}\left(\pi / 2-\beta,-h^{2}\right)=(-1)^{m} c e_{2 m}\left(\beta, h^{2}\right) .
$$

The integrand of the last integral is odd so that this term is zero; hence

$$
I_{2 m}=\int_{0}^{\infty} \cos (\lambda \cos \alpha \sinh t) d t \int_{0}^{\pi} c e_{2 m}\left(\beta,-\frac{\lambda^{2}}{4}\right) \cos (\lambda \cos \beta \sinh t) d \beta .
$$

Now $[6$, p. 187] states

$$
C e_{2 m}\left(z, \frac{\lambda^{2}}{4}\right)=\left[A_{0}^{(2 m)} \pi\right]^{-1} c e_{2 m}\left(0, \frac{\lambda^{2}}{4}\right) \int_{0}^{\pi} \cos (\lambda \sin u \sinh z) c e_{2 m}\left(u, \frac{\lambda^{2}}{4}\right) d u
$$

and therefore

$C e_{2 m}\left(z, \frac{\lambda^{2}}{4}\right)=\left[A_{0}^{(2 m)} \pi\right]^{-1} c e_{2 m}\left(0, \frac{\lambda^{2}}{4}\right)(-1)^{m} \int_{0}^{\pi} \cos (\lambda \cos \beta \sinh z) c e_{2 m}\left(\beta,-\frac{\lambda^{2}}{4}\right) d \beta$

for we may substitute $u=\pi / 2-\beta$ and reset the range of integration to $(0, \pi)$ since the integrand has period $\pi$. Thus (3.3) becomes

$$
I_{2 m}=A_{0}^{(2 m)} \pi \cdot\left[c e_{2 m}\left(0, \frac{\lambda^{2}}{4}\right)\right]^{-1}(-1)^{m} \int_{0}^{\infty} C e_{2 m}\left(t, \frac{\lambda^{2}}{4}\right) \cos (\lambda \cos \alpha \sinh t) d t .
$$

Now consider

$$
d_{r}=\frac{2}{\pi} \int_{0}^{\pi} I_{2 m}(\alpha) c e_{r}\left(\alpha,-\frac{\lambda^{2}}{4}\right) d \alpha
$$


From (3.5), and an inversion in the order of integrations again,

$d_{r}=2 A_{0}^{(2 m)}\left[c e_{2 m}\left(0, \frac{\lambda^{2}}{4}\right)\right]^{-1}(-1)^{m} \int_{0}^{\infty} C e_{2 m}\left(t, \frac{\lambda^{2}}{4}\right) d t \int_{0}^{\pi} c e_{r}\left(\alpha,-\frac{\lambda^{2}}{4}\right)$

$\cdot \cos (\lambda \cos \alpha \sinh t) d \alpha$.

Let us deal first with $r$ odd; then the inner integral of the right side of (3.6) becomes $\int_{0}^{\pi} c e_{2 s+1}\left(\alpha,-\frac{\lambda^{2}}{4}\right) \cos (\lambda \cos \alpha \sinh t) d \alpha$

$$
=(-1)^{s} \int_{-\pi / 2}^{\pi / s} s e_{2 s+1}\left(\alpha_{1}, \frac{\lambda^{2}}{4}\right) \cos \left(\lambda \sin \alpha_{1} \sinh t\right) d \alpha_{1},
$$

by substituting $\alpha_{1}=\pi / 2-\alpha$, which is zero since the integrand is odd in $\alpha_{1}$.

For $r$ even, $2 s$ say, we can use (3.4) again and obtain

$$
d_{2 s}=2 \pi(-1)^{m+s} A_{0}^{(2 m)} A_{0}^{(2 s)}\left[c e_{2 m}\left(0, \frac{\lambda^{2}}{4}\right) c e_{2 s}\left(0, \frac{\lambda^{2}}{4}\right)\right]^{-1} \int_{0}^{\infty} C e_{2 m}\left(t, \frac{\lambda^{2}}{4}\right) C e_{2 s}\left(t, \frac{\lambda^{2}}{4}\right) d t \text {. }
$$

We now evaluate

$$
J_{m s}=\int_{0}^{\infty} C e_{2 m}\left(t, \frac{\lambda^{2}}{4}\right) C e_{2 s}\left(t, \frac{\lambda^{2}}{4}\right) d t
$$

From [6, p. 187], we have

$$
C e_{2 m}\left(z, \frac{\lambda^{2}}{4}\right)=c e_{2 m}\left(\frac{\pi}{2}, \frac{\lambda^{2}}{4}\right)\left[\pi A_{0}^{(2 m)}\right]^{-1} \int_{0}^{\pi} \cos (\lambda \cos u \cosh z) c e_{2 m}\left(u, \frac{\lambda^{2}}{4}\right) d u
$$

and by the same procedures as those used for (3.4) we can deduce that

$C e_{2 m}\left(z, \frac{\lambda^{2}}{4}\right)=(-1)^{m} c e_{2 m}\left(\frac{\pi}{2}, \frac{\lambda^{2}}{4}\right)\left[\pi A_{0}^{(2 m)}\right]^{-1} \int_{0}^{\pi} \cos (\lambda \sin u \cosh z) c e_{2 m}\left(u,-\frac{\lambda^{2}}{4}\right) d u$.

Thus if we substitute this result in (3.8) and invert the order of integration we have $J_{m s}=(-1)^{m} c e_{2 m}\left(\frac{\pi}{2}, \frac{\lambda^{2}}{4}\right)\left[\pi A_{0}^{(2 m)}\right]^{-1} \int_{0}^{\pi} c e_{2 m}\left(u,-\frac{\lambda^{2}}{4}\right) d u \int_{0}^{\infty} C e_{2 s}\left(t, \frac{\lambda^{2}}{4}\right)$ $\cdot \cos (\lambda \sin u \cosh t) d t$.

Now [6, p. 199] states that

$F e k_{2 s}\left(z,-\frac{\lambda^{2}}{4}\right)=(-1)^{s} \cdot c e_{2 s}\left(\frac{\pi}{2}, \frac{\lambda^{2}}{4}\right)\left[\pi A_{0}^{(2 s)}\right]^{-1} \int_{0}^{\infty} \exp (-\lambda \sinh z \cosh t)\left(e_{2 s}\left(t, \frac{\lambda^{2}}{4}\right) d t\right.$, and with $z$ replaced by $i z$, we have $F e k_{2 s}\left(i z,-\frac{\lambda^{2}}{4}\right)=(-1)^{s} c e_{2 \cdot}\left(\frac{\pi}{2}, \frac{\lambda^{2}}{4}\right)\left[\pi A_{0}^{(2 s)}\right]^{-1} \int_{0}^{\infty} \exp (-\lambda i \sin z \cosh t) C e_{2 *}\left(t, \frac{\lambda^{2}}{4}\right) d t$

But also 
$F e k_{2 s}\left(i z,-\frac{\lambda^{2}}{4}\right)=\frac{F e k_{2 s}\left(0,-\frac{\lambda^{2}}{4}\right)}{C e_{2 s}\left(0,-\frac{\lambda^{2}}{4}\right)} C e_{2 s}\left(i z,-\frac{\lambda^{2}}{4}\right)+\frac{F e k_{2 s}^{\prime}\left(0,-\frac{\lambda^{2}}{4}\right)}{F e_{2 s}^{\prime}\left(0,-\frac{\lambda^{2}}{4}\right)} F e_{2 s}\left(i z,-\frac{\lambda^{2}}{4}\right)$

from [6, p. 249] with $z$ replaced by $i z$.

All terms on the right-hand side of (3.12) are real with the exception of $\mathrm{Fe}_{2 s}\left(i z,-\lambda^{2} / 4\right)$ which is purely imaginary. Therefore if we compare the real parts of the right-hand sides of (3.12) and (3.11) we have

$$
\begin{aligned}
(-1)^{s} c e_{2 s}\left(\frac{\pi}{2}, \frac{\lambda^{2}}{4}\right)\left[\pi A_{0}^{(2 s)}\right]^{-1} \int_{0}^{\infty} \cos (\lambda \sin z \cosh t) C e_{2 s}\left(t, \frac{\lambda^{2}}{4}\right) d t & \\
= & \frac{F e k_{2 s}\left(0,-\frac{\lambda^{2}}{4}\right)}{C e_{2 s}\left(0,-\frac{\lambda^{2}}{4}\right)} C e_{2 s}\left(i z,-\frac{\lambda^{2}}{4}\right) .
\end{aligned}
$$

From the definition of the $C e_{n}{ }^{\prime} \mathrm{s}$,

$$
C e_{2 s}\left(i z,-\lambda^{2} / 4\right)=c e_{2 s}\left(z,-\lambda^{2} / 4\right)
$$

and

$$
C e_{2 s}\left(0,-\lambda^{2} / 4\right)=c e_{2 s}\left(0,-\lambda^{2} / 4\right)=(-1)^{s} c e_{2 s}\left(\pi / 2, \lambda^{2} / 4\right) .
$$

These last three results may be substituted into the inner integral of (3.10) and after some elementary manipulations we obtain

$J_{m s}=(-1)^{m} \frac{c e_{2 m}\left(\frac{\pi}{2}, \frac{\lambda^{2}}{4}\right)}{\left[c e_{2 s}\left(\frac{\pi}{2}, \frac{\lambda^{2}}{4}\right)\right]^{2}} A_{0}^{(2 s)}\left[A_{0}^{2 m}\right]^{-1} F e k_{2 s}\left(0,-\frac{\lambda^{2}}{4}\right) \int_{0}^{\pi} c e_{2 m}\left(u,-\frac{\lambda^{2}}{4}\right) c e_{2 s}\left(u,-\frac{\lambda^{2}}{4}\right) d u$

and since the $\mathrm{ce}_{n}$ 's are orthogonal on $(0, \pi)$ we have

$$
\begin{aligned}
J_{m m} & =(-1)^{m}\left[c e_{2 m}\left(\frac{\pi}{2}, \frac{\lambda^{2}}{4}\right)\right]^{-1} F e k_{2 m}\left(0,-\frac{\lambda^{2}}{4}\right) \frac{\pi}{2} \\
J_{m s} & =0, \quad m \neq s .
\end{aligned}
$$

This result, which is quite unremarkable, does not appear in the literature and some observations are relevant. We can arrive at $(3.15 \mathrm{~b})$ by an application of those methods used for Sturm-Liouville problems; however, this has the disadvantage that we do not evaluate $J_{m m}$ by such a process whereas in the above procedure we obtain $J_{m s}$ for both $m=s$ and $m \neq s$. An interesting detail which does emerge from the Sturm-Liouville approach is that whereas the even parity functions, $C e_{2 n}\left(t, \lambda^{2} / 4\right)$, are mutually orthogonal (as are the odd parity functions) those with even parity are not orthogonal to those of odd parity. This follows because the functions of odd parity do not have quite the same behaviour at infinity as those of even parity. Exactly similar results may be obtained for

$$
\int_{0}^{\infty} S e_{i}\left(z, \frac{\lambda^{2}}{4}\right) S e_{k}\left(z, \frac{\lambda^{2}}{4}\right) d z .
$$


We now apply (3.15) to (3.7) and obtain $d_{2 s}=0, s \neq m$, and

$$
d_{2 m}=(-1)^{m}\left[\pi A_{0}^{(2 m)}\right]^{2}\left[c e_{2 m}\left(0, \frac{\lambda^{2}}{4}\right)\right]^{-2}\left[c e_{2 m}\left(\frac{\pi}{2}, \frac{\lambda^{2}}{4}\right)\right]^{-1} F e k_{2 m}\left(0,-\frac{\lambda^{2}}{4}\right) \text {. }
$$

It follows from these results that

$$
I_{2 m}=d_{2 m} \cdot c e_{2 m}\left(\alpha,-\frac{\lambda^{2}}{4}\right)
$$

A comparison of this result with (2.11) shows that $d_{2 m}$ must equal $-\pi F e k_{2 m}(0)\left[F e k_{2 m}^{\prime}(0)\right]^{-1}$, and this is indeed true, for, combining the formulae (13) p. 249, and (3) p. 368 of [6], we find that

$$
-\pi \cdot\left[F e k_{2 m}^{\prime}(0)\right]^{-1}=(-1)^{m}\left[\pi A_{0}^{(2 m)}\right]^{2}\left[c e_{2 m}\left(0, \frac{\lambda^{2}}{4}\right)\right]^{-2}\left[c e_{2 m}\left(\frac{\pi}{2}, \frac{\lambda^{2}}{4}\right)\right]^{-1} .
$$

The problem is completed when we have dealt with the case of $p$ odd. We conclude by observing that the methods used are identical to those given so far and they will therefore be omitted.

Acknowledgments. This problem was suggested to me by Professor A. F. Pillow whom I would like to thank for his valuable comments on the problem. I would also like to express thanks to Dr. V. G. Hart for many helpful discussions and comments.

\section{References}

[1] V. L. Rvachev, The pressure on an elastic half space of a beam which has the form of a strip, Prikl. Mat. Mekh. 20, 248-253 (1956)

[2] A. E. Gill, Forced convection, M.Sc. Thesis, University of Melbourne, 1960

[3] G. E. Latta, The solution of a class of integral equations, J. Rational Mech. Anal. 5, 821-834 (1956)

[4] C. E. Pearson, On the finite strip problem, Quart. Appl. Math. 15, pp. 203-208 (1958)

[5] T. Lundgren and D. Chiang, Solution of a class of singular integral equations, Quart. Appl. Math. 24, 303-313 (1967)

[6] N. W. McLachlan, Theory and application of Mathieu functions, Oxford University Press, 1951

[7] J. Meixner and F. W. Schäfke, Mathieusche Funktionen und Sphäroidfunktionen, Springer-Verlag, Berlin, 1954 